\title{
Hadronic interactions and Cosmic Ray mass composition studied by ARGO-YBJ
}

\author{
Antonio SURDO* \\ INFN - Sezione di Lecce, Italy \\ E-mail: surdo@le.infn.it \\ on behalf of the ARGO-YBJ Collaboration
}

The ARGO-YBJ detector layout, features and location at high altitude $\left(\sim 606 \mathrm{~g} / \mathrm{cm}^{2}\right.$ of atmospheric depth) offer a unique chance for a detailed study of several characteristics of the hadronic component of the Cosmic Rays in the $10^{12}-10^{15} \mathrm{eV}$ energy range. Indeed, the analog readout of the RPC (Resistive Plate Chamber) signals provides a powerful tool to study, with unprecedented resolution, the distribution of the charged particles of extensive air showers (EAS) down to few meters from the axis, thus allowing to describe its shape in detail and to estimate the shower age at the detection level.

These features make feasible, for the first time, mass composition studies with an EAS detector in an energy region where the comparison with direct measurements is now possible. Moreover, new inputs to the hadronic interaction models, currently used for the analysis of the cosmic ray flux and origin up to the highest energies, can be obtained.

The European Physical Society Conference on High Energy Physics

18-24 July, 2013

Stockholm, Sweden

\footnotetext{
* Speaker.
} 

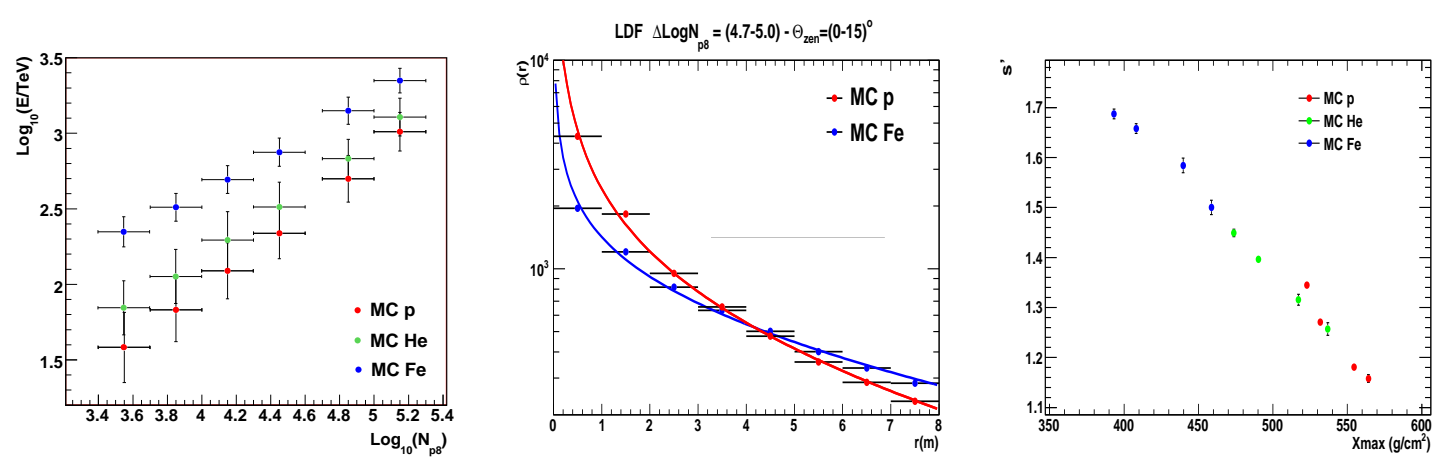

Figure 1: Left panel: Average primary energy for $\mathrm{p}, \mathrm{He}, \mathrm{Fe}$ initiated $\mathrm{MC}$ showers and several $N_{p 8}$ intervals. Central panel: Reconstructed average LDF for MC p and Fe showers $\left(N_{p 8}=10^{4.7}-10^{5.0}\right)$, fitted by Eq.2.1. Right panel: Age parameter $s^{\prime}$ from fits of LDF of MC p, He and Fe samples (in each $N_{p 8}$ bin) vs $\left\langle X_{\max }\right\rangle$.

\section{The ARGO-YBJ experiment}

The ARGO-YBJ detector [1] is a full coverage extensive air shower (EAS) array made by a single layer of Resistive Plate Chambers (RPCs) for $\gamma$-astronomy and cosmic ray (CR) studies. The array was installed in the Cosmic Ray Laboratory of YanBaJing (Tibet, China), at an altitude of $4300 \mathrm{~m}$ above sea level (vertical atmospheric depth $\sim 606 \mathrm{~g} / \mathrm{cm}^{2}$ ). The detector layout and features (full-coverage, space pixel size, time resolution) provided a detailed digital event imaging and reconstruction, while the RPC analog charge readout allowed to extend the operating range up to $\sim \mathrm{PeV}$ primary proton energies and measure the ground particle densities very close to the shower core. This implies a unique chance for the measurement of the p-air cross section in the 0.1-1 PeV range and for the study of several characteristics of the primary $\mathrm{CR}$ hadronic component.

\section{Data analysis and results}

An EAS array by itself cannot measure directly the shower development stage, but simply the particle density distribution at ground as a function of the core distance (LDF). Anyway, the detailed LDF study is expected to provide information useful to select intervals of $X_{\max }$ (depth of the cascade maximum size), important for measuring p-air cross section by the flux attenuation method [2]. In turn, the shower development stage as observed at detection level, depends on the energy $E$ of the interacting primary, while, for fixed $E$, it depends on the primary type. Then, the combined use of shower $E$ and age estimations ensures a sensitivity to the primary nature.

To perform this analysis, several samples of simulated showers by different primaries ( $\mathrm{p}, \mathrm{He}$ and Fe nuclei) were generated and submitted to a full detector simulation. Monte Carlo (MC) events triggering the analog RPC readout (with reconstructed core in a $64 \times 64 \mathrm{~m}^{2}$ detector internal area and $\theta_{\text {zenith }}<15^{\circ}$ ) were then analyzed to find a suitable estimator of the primary CR energy $E$. The number of particles detected within $8 \mathrm{~m}$ from the shower axis $\left(N_{p 8}\right)$ resulted well correlated to $E$, not biased by the finite detector size and lightly affected by shower to shower fluctuations. In Fig.1 (left panel), such correlation is shown for p, He and Fe initiated showers. 

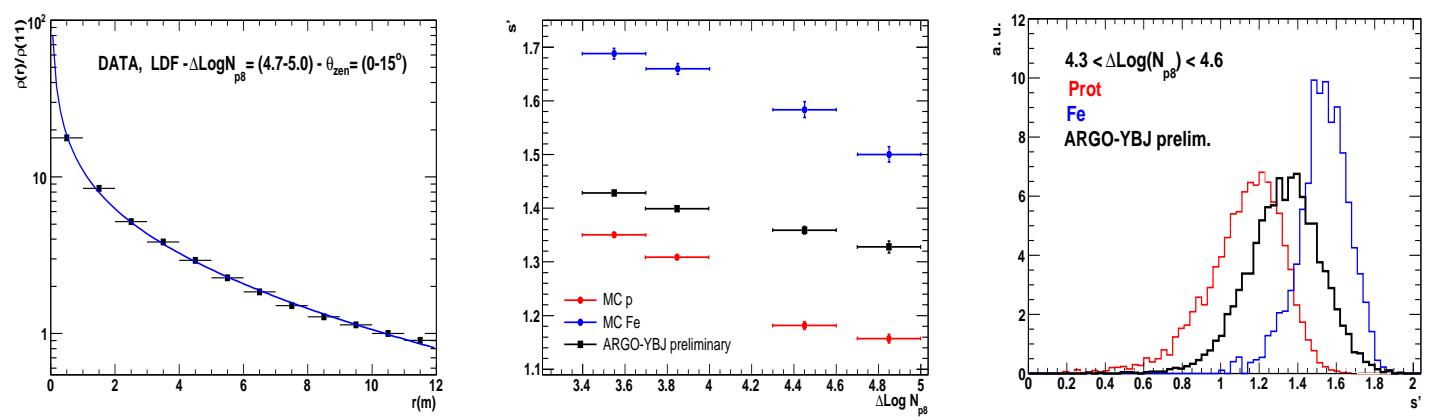

Figure 2: Left panel: Average LDF for real ARGO-YBJ events with $N_{p 8}=10^{4.7}-10^{5.0}$. The fit with the NKG-like function is also shown. Central panel: Age parameter $s^{\prime}$ from the fits of average LDFs of real ARGO-YBJ data and MC $\mathrm{p}$ and Fe induced showers, in four $N_{p 8}$ bins. Right panel: Age parameter $s^{\prime}$ from event-by-event LDF fits for real ARGO-YBJ data and MC p and Fe induced showers, in one of the $N_{p 8}$ bins.

The reconstructed LDFs (up to $\sim 10 \mathrm{~m}$ from the core), for different $N_{p 8}$ (i.e. energy) intervals and shower initiating primaries, have been fitted with the following NKG-like function:

$$
\rho(r)=A \times\left(\frac{r}{r_{0}}\right)^{s^{\prime}-2}\left(1+\frac{r}{r_{0}}\right)^{s^{\prime}-4.5}
$$

being $A$ and $s^{\prime}$ free parameters. Fig.1 (central panel) shows such fits for $\mathrm{p}$ and Fe induced showers.

It is found that $s^{\prime}$ plays the role of the 'lateral age' parameter, thus reflecting the shower development stage. This implies that $s^{\prime}$ can help to constrain the shower $X_{\max }$ position, as demonstrated by Fig. 1 (right panel), where the $s^{\prime}$ values from the LDF fit, for each primary and $N_{p 8}$ interval, vs the average value of $X_{\max }$ distribution from MC simulation, are shown. That plot expresses an important universality of the LDF of detected EAS particles in terms of the lateral shower age, since the shape parameter $s^{\prime}$ depends only on the development stage of the shower, independently from the nature of the primary particle and energy. Moreover, $s^{\prime}$ from the LDF fit very close to the shower axis, together with the measurement of the truncated size $N_{p 8}$, can give information on the primary particle nature, thus making possible the study of primary mass composition (see [3]).

Also for experimental data, the ARGO-YBJ LDF distributions appear properly described by the function 2.1, as shown in Fig.2 (left panel) in the case of an exemplifying $N_{p 8}$ interval. The $s^{\prime}$ values from experimental data are reported in Fig.2 (central panel) together with the corresponding fit results from MC simulations for different primaries. In spite of the preliminary nature of this result, we observe that the data points lie between the expectations from extreme pure compositions.

The effects of intrinsic shower-to-shower fluctuations on the sensitivity to mass composition can be avaluated from the single event LDF fits. The result in terms of $s^{\prime}$ distributions is shown in Fig.2 (right panel) for ARGO-YBJ data and simulated p and Fe initiated showers, in one $N_{p 8}$ bin.

\section{References}

[1] G. Aielli et al., NIM A, 562, 92 (2006)

[2] G. Aielli et al., Phys. Rev. D, 80, 092004 (2009)

[3] A. D’Amone et al., Proc. 33rd ICRC (2013), paper id. 779. 